\title{
Monte Carlo Simulations of Weapon Effectiveness Using Pk Matrix and Carleton Damage Function
}

\author{
Pawat Chusilp, Weerawut Charubhun, and Pattadon Koanantachai
}

\begin{abstract}
Carleton damage function has been commonly utilized to describe the weapon-target interaction in the weapon effectiveness analyses. This function is simplified from the actual weapon lethality data and these simplifications can affect the analysis results. This paper investigates the difference between results of Monte Carlo simulations to determine the probability of target damage that utilize the Carleton damage function and the results of the same simulations that utilize a non-simplified probability of kill (Pk) matrix. A problem of multiple shots of an unguided artillery weapon against an area target was chosen as a case study. Two sets of Monte Carlo simulations to determine the probability of damage on a target were performed for several numbers of shots and target sizes. The first set of simulations utilized the Pk matrix while the second set utilized the Carleton damage function. Statistical analyses were performed. It was suggested that there was difference between the results of two sets of simulations but the effect size was small.
\end{abstract}

Index Terms-Damage function, probability of damage, Monte Carlo simulation, weapon effectiveness.

\section{INTRODUCTION}

Monte Carlo methods have been employed in the field of weapon effectiveness to determine the probability of target damage when there are no close form solutions available. A Monte Carlo simulation to determine the probability of damage on an area target inflicted by artillery weapons normally comprises several thousand runs. In each run, the impact points of all shots are randomized in accordance with the weapon delivery accuracy. Then the probability of damage inflicted by the weapons on the targets in each run can be evaluated per weapon lethality data, which describe the interaction between a specific weapon and a specific type of targets. These weapon lethality data are normally determined by testing or numerical simulation. They are often presented in the form of Pk (Probability of kill) matrix that describes $\mathrm{Pk}$ value at various distances around the impact point. This format may be too complicated for computing in the operations so the lethality data are approximated and replaced by closed form damage functions.

Several damage functions have been described in many literatures and text books [1]-[5]. Two most widely used damage functions are the cookie cutter function and the Carleton function. The cookie cutter function simply assumes that fraction of the targets that lies inside the weapon lethal

Manuscript received May 2, 2014; revised July 2, 2014.

Pawat Chusilp, Weerawut Charubhun, and Pattadon Koanantachai are with Defence Technology Institute, 47/433 Changewattana Road, Pakkred, Nonthaburi 11120, Thailand (e-mail: pawat.c@dti.or.th, weerawut.c@dti.or.th,pattadon.k@dti.or.th). area will be completely destroyed while the rest will receive no damage. The Carleton function assumes Gaussian distribution of Pk value at any distance from the impact point. An example of recent research works that employed these damage functions in Monte Carlo simulations to analyze weapon effectiveness is Anderson [6]. In his work, Monte Carlo simulations were performed to determine the probability of damage inflicted on a point and an area target by a single and a stick of air-to-surface weapons. The simulations utilized the rectangular cookie cutter and the Carleton function. The results from the Monte Carlo simulations were compared to the results from JMEM (Joint Munitions Effectiveness Manuals) method, which is a standardized methodology developed by JTCG/ME (Joint Technical Coordinating Group for Munitions Effectiveness) in the United States of America [7].

In most cases of fragmentation weapons against personnel targets, the Carleton function is apparently more realistic than the cookie cutter function in representing the weapon lethality. However, the Carleton function still differs from the non-simplified Pk matrix and it can possibly affects the results of Monte Carlo simulations. The objective of this paper is to investigate whether the results of Monte Carlo simulations that utilize the Carleton function differ from the simulations that utilize a non-simplified Pk matrix. The focus of this paper is on the problem of multiple shots of unguided artillery weapon against a uniform value area target.

From the next section, the paper is outlined as follows. Section II briefly explains the calculation steps in a Monte Carlo simulation to determine the probability of damage for multiple shots against area targets. Section III describes the Carleton damage function and how its parameters are determined. Section IV describes a case study. The results are presented and discussed in Section V. Section VI summarizes the paper.

\section{Monte CARlo Simulation to Determine the PROBABILITY OF DAMAGE}

A Monte Carlo method can refers to a computing technique that determines results from many repeating random sampling. Scientists who involved in the United States nuclear project during World War II are often regarded as the inventors of the Monte Carlo method [8], [9]. The method is very useful when it is difficult or impossible to obtain closed form solutions. This method has been successfully applied to many scientific problems including the weapon effectiveness and target coverage topics. In most applications, Monte Carlo simulations are carried out by computer programs for computing speed. 
Fig. 1 illustrates the process of a Monte Carlo simulation to determine the probability of damage on targets inflicted by multiple shots of weapons. First, the target geometry must be defined. Next a boundary box that encloses all targets is created and grid points are generated uniformly inside the boundary box. These grid points serve as integration points to calculate the damage value of the whole target. Refinement of the grid depends on the shapes of targets and complication of the damage function. Then the Monte Carlo simulation is performed for several runs.

In each Monte Carlo run, the impact points of all shots are randomized in accordance with the weapon delivery accuracy. The probability of damage at each grid point can be determined by (1) and the probability of damage of the whole target in this run is determined by (2). after all runs have been performed, the result of the simulation can be calculated from the mean of results in all runs, as given in (3). Note that the probability of damage normally ranges from 0 (unharmed) to 1.0 (completely destroyed).

$$
\begin{gathered}
d_{i}=1-\left(1-p_{1}\right)\left(1-p_{2}\right)\left(1-p_{3}\right) \ldots\left(1-p_{s}\right) \\
d_{\text {total }}=\frac{\sum_{i=1}^{M} d_{i}}{M} \\
D=\frac{\sum_{k=1}^{N} d_{\text {total }, k}}{N}
\end{gathered}
$$

where

$$
\begin{aligned}
& d_{i}=\text { probability of damage at grid point } i^{\text {th }} \\
& p_{s}=\text { Pk value at grid point } i^{\text {th }} \text { done by } s^{\text {th }} \text { shot } \\
& d_{\text {total }}=\text { probability of damage on target in each run } \\
& d_{\text {total, },}=\text { probability of damage on target in } k^{\text {th }} \text { run } \\
& D=\text { probability of damage on target } \\
& M=\text { total number of grid points inside target } \\
& N=\text { total number of runs in a simulation }
\end{aligned}
$$

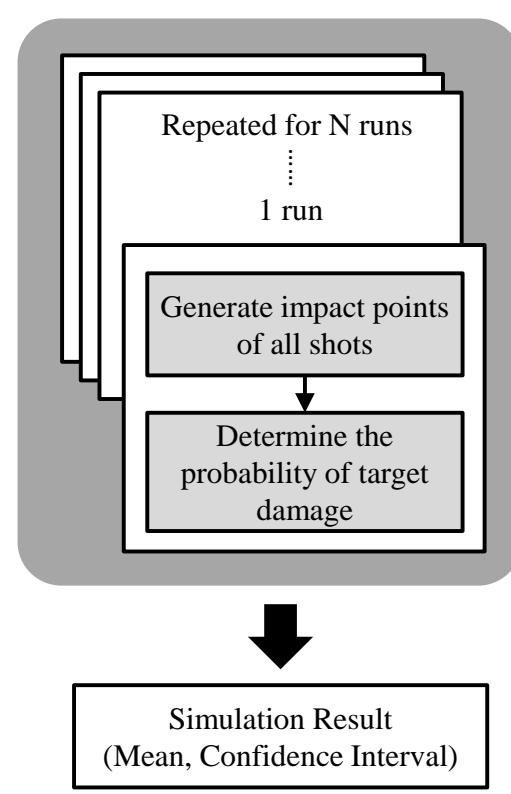

Fig. 1. Overall process of a Monte Carlo simulation.
The validity of the results of Monte Carlo simulations depends on the number of runs performed in the simulation [10], [11]. A confidence interval $C I$ of the simulation result is given in (4).

$$
C I=\left(D-\frac{Z_{1-\alpha / 2} s t d}{\sqrt{N}}, D+\frac{Z_{1-\alpha / 2} s t d}{\sqrt{N}}\right)
$$

where

$$
\begin{aligned}
& Z_{1-\alpha / 2}=Z \text { value at } 1-\alpha / 2 \text { confidence level } \\
& s t d=\text { standard deviation of samples }
\end{aligned}
$$

\section{CARleton DAmage Function}

In the Carleton damage function, a bivariate Gaussian distribution of $\mathrm{Pk}$ value in range and deflection direction is assumed. The Pk value from one shot impacted at a point $(x, y)$ is given in (5). This $\mathrm{Pk}$ value is used in (1).

$$
p(x, y)=\exp \left(-\left(\frac{x}{R_{x}}\right)^{2}-\left(\frac{y}{R_{y}}\right)^{2}\right)
$$

where

$p(x, y)=$ probability of damage at point $(x, y)$

$x=$ distance in range from impact point

$y=$ distance in deflection from impact point

$R^{x}=$ constant related to weapon lethality in range direction

$R_{y}=$ constant related to weapon lethality in deflection direction

\section{CASE STUdY}

\section{A. Description}

A problem of firing multiple shots of an artillery weapon against an area target was chosen as a case study. Two sets of Monte Carlo simulations to determine the probability of damage on a target were performed. The first set utilized a non-simplified $\mathrm{Pk}$ matrix, which is treated as a high fidelity model. The second set utilized the Carleton function. Each sets were performed for 10, 20, 30 shots and 2 targets of the same shape and proportion but different size. A set of impact points was generated for all runs and used for both sets of the simulations. So the difference between the results from the first set and the second set could be determined. In total, 6 Monte Carlo simulations have been performed for each simulation set. Each Monte Carlo simulation comprised 10000 runs. The simulations were carried out in a MATLAB ${ }^{\circledR}$ program.

Because the result of a Monte Carlo simulation is in fact the mean of results in all runs, a paired t-test can be conducted to test the difference between the results of two Monte Carlo simulations that employed $\mathrm{Pk}$ matrix and the Carleton function on the same target and same number of shots using the same set of pre-generated impact points. The comparison is similar to a paired observation that the same sample group (same impact points) receives both treatments ( $\mathrm{Pk}$ matrix and the Carleton function) and has a number of 
pairs of observations (probability of damage on target in each run). Totally, 6 paired t-tests have been performed in the case study.

Let $\mu_{1}$ be the result of a Monte Carlo simulation that employs the $\mathrm{Pk}$ matrix, $\mu_{2}$ be the result of a Monte Carlo simulation that employs the Carleton function, $\mu_{d}$ be the mean of the difference between the paired results, which is equal to $\mu_{1}-\mu_{2}$ since the observations are paired. The following null hypothesis $H_{0}$ and alternative hypothesis $\mathrm{H}_{1}$ were tested at the 0.01 significance level.

$$
\begin{array}{ll}
H_{0}: & \mu_{d}=0 \\
H_{1}: & \mu_{d} \neq 0
\end{array}
$$

\section{B. Weapon}

A weapon chosen for the case study was a research fragmentation warhead. Fig. 2 presents the contour of the $\mathrm{Pk}$ matrix of the weapon when the detonation point is at $(0,0)$. Only half of the figure is presented because the figure is symmetrical about the range axis. The lethal area $A_{L}$ of the warhead was calculated by (6), as defined in several literatures [3], [5], [6]. Note that $p(x, y)$ is the probability of kill at a point $(x, y)$, which is used in (1). Because the resolution of the grid could affect the value of the lethal area calculated by (6), a grid refinement study was carried out. It was found that the value of lethal area approached $6192 \mathrm{~m}^{2}$ and a grid size of $0.6 \mathrm{~m}$ is appropriate for the simulations.

$$
\begin{aligned}
A_{L} & =\int_{-\infty-\infty}^{\infty} \int^{\infty} p(x, y) d x d y \\
& \approx \sum_{y=-\infty}^{\infty} \sum_{x=-\infty}^{\infty} p(x, y) \Delta x \Delta y
\end{aligned}
$$

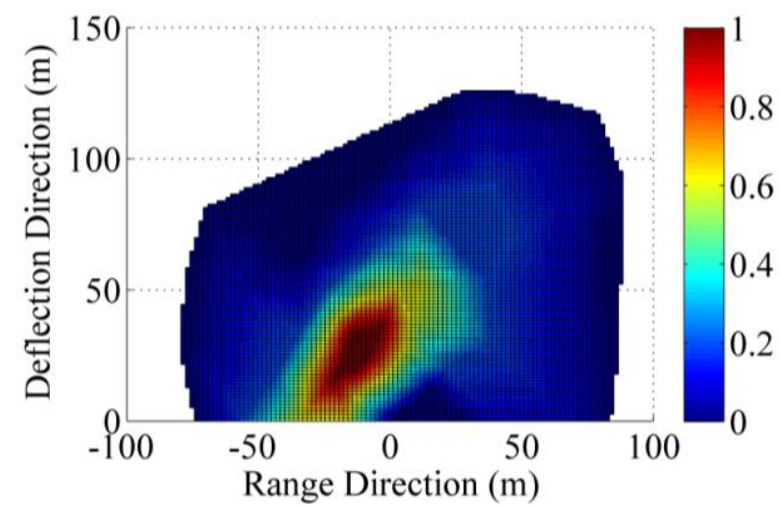

Fig. 2. Lethality of the warhead by Pk matrix.

\section{Parameters of Carleton Function}

The constants of the Carleton damage function could be determined by applying a regression technique [12] on the data in the Pk matrix. But for the two parameters Carleton function employed in this paper, $R_{x}$ and $R_{y}$ were simply determined by a guideline described in Driels [6]. The key points are that the lethal area must be conserved and a ratio $a$ between $R_{x}$ and $R_{y}$ is a function of impact angle $I$ as given in (7). In all simulations, $R_{x}=24.87 \mathrm{~m}$ and $R_{y}=80.97 \mathrm{~m}$ were used.

$$
a=\max (0.3,1-0.8 \cos I)
$$

In the simulations, all grid points located outside an ellipse, of which the center is at the impact point and the major and minor axes are $6 R_{x}$ and $6 R_{y}$, were neglected because the $\mathrm{Pk}$ value would be very small. A grid refinement study was also conducted to ensure that the lethal area, as given in (6), calculated by the Carleton function is equal the lethal area calculated by $\mathrm{Pk}$ matrix. In addition, it was found that the Carleton function actually required much coarser grid size, and hence much less calculation time. But to eliminate any effect on the results caused by different grid size, the same grid size of $0.6 \mathrm{~m}$ was used in both simulations that employed $\mathrm{Pk}$ matrix and the Carleton function.

\section{Targets}

The targets in the case study were a rectangular area orientated at $30^{\circ}$ from the range axis as shown in Fig. 3. The target was assumed to be uniformly valued. All shots were aimed at the centroid of the target area. The simulations were performed on two targets that have similar shape and proportion but different size. The area of the first target is $15000 \mathrm{~m}^{2}$, which is approximately 2.5 times of the weapon lethal area. The area of the second target is $7500 \mathrm{~m}^{2}$, which is 1.2 times of the weapon lethal area.

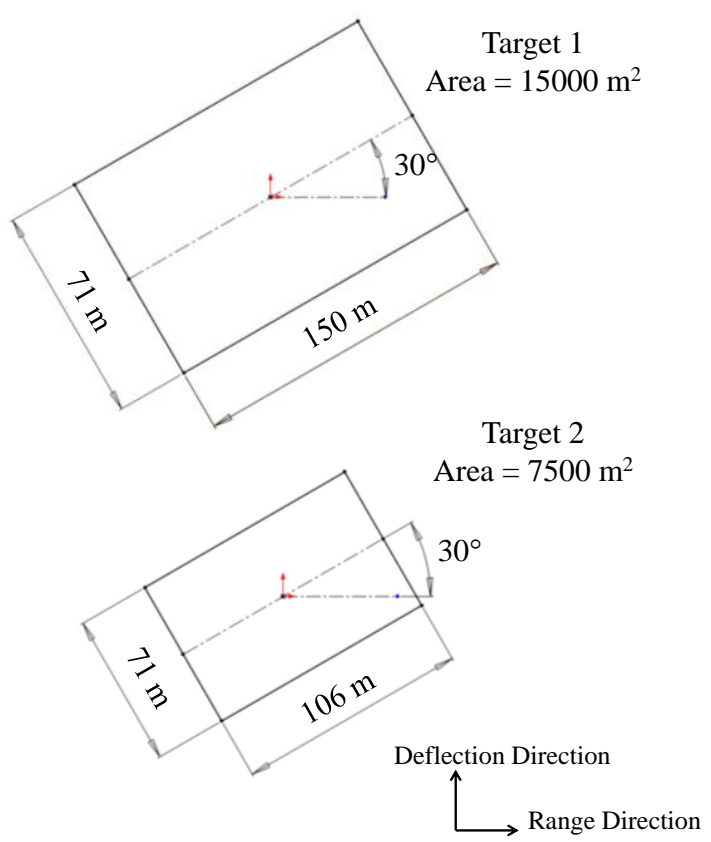

Fig. 3. Targets.

\section{E. Delivery Errors}

The impact points of all shots were generated randomly in accordance with REP (Range Error Probable) and DEP (Deflection Error Probable). Two types of errors were considered. They are bias errors and dispersion errors. The bias errors affect all shots equally while the dispersion errors affect each round individually. Both types of errors were assumed to be bivariate Gaussian distributed. In this case study, we assumed following REP and DEP of bias and dispersion errors:

$$
\begin{array}{lcc}
\text { Bias Error: } & R E P=30 \mathrm{~m} \quad D E P=30 \mathrm{~m} \\
\text { Dispersion Error: } & R E P=150 \mathrm{~m} \quad D E P=50 \mathrm{~m}
\end{array}
$$




\section{RESUlts}

An example of the impact points generated for one Monte Carlo run is presented in Fig. 4. The yellowed area indicates the target area and the red X marker denotes the impact point and the number denotes the shot id number. Fig. 5 shows the mean of the results from the first run to the current run monitored during the simulation. In all simulations, the result approached the final value within 3000 runs approximately.

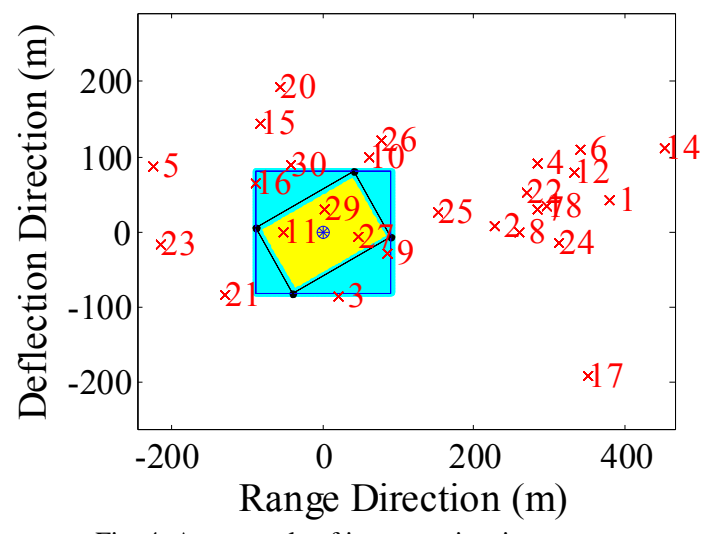

Fig. 4. An example of impact points in one run.

$\begin{array}{rr}-30 \text { shots, Pk matrix } & ---30 \text { shots, Carleton Fnc } \\ -20 \text { shots, Pk matrix } & ---20 \text { shots, Carleton Fnc } \\ -10 \text { shots, Pk matrix } & ---10 \text { shots, Carleton Fnc }\end{array}$

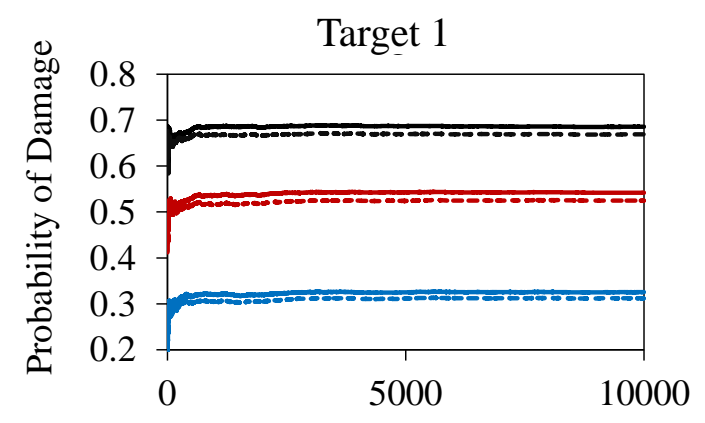

Number of Runs

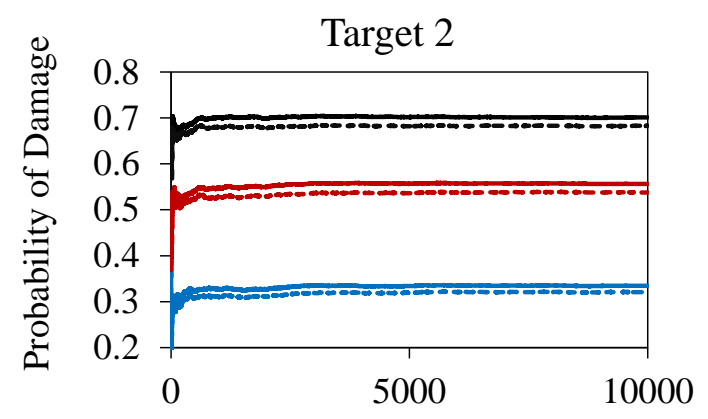

Number of Runs

Fig. 5. Simulation results monitored during the calculation.

The results of all simulations from 10000 runs are presented in Fig. 6 and the difference between the results is summarized in Table I and Table II. Overall, the results of the simulations that utilized the Carleton function were 2 to $4 \%$ lower than the results of the simulations that utilized $\mathrm{Pk}$ matrix in all cases of target sizes and number of shots. The time required for the simulations that employed $\mathrm{Pk}$ matrix was much greater than the simulations that employed the Carleton function. But calculation time also depends on the algorithms, which is not the focus of this paper.

\section{$\square$ Use Pk Matrix $\square$ Use Carleton Function}
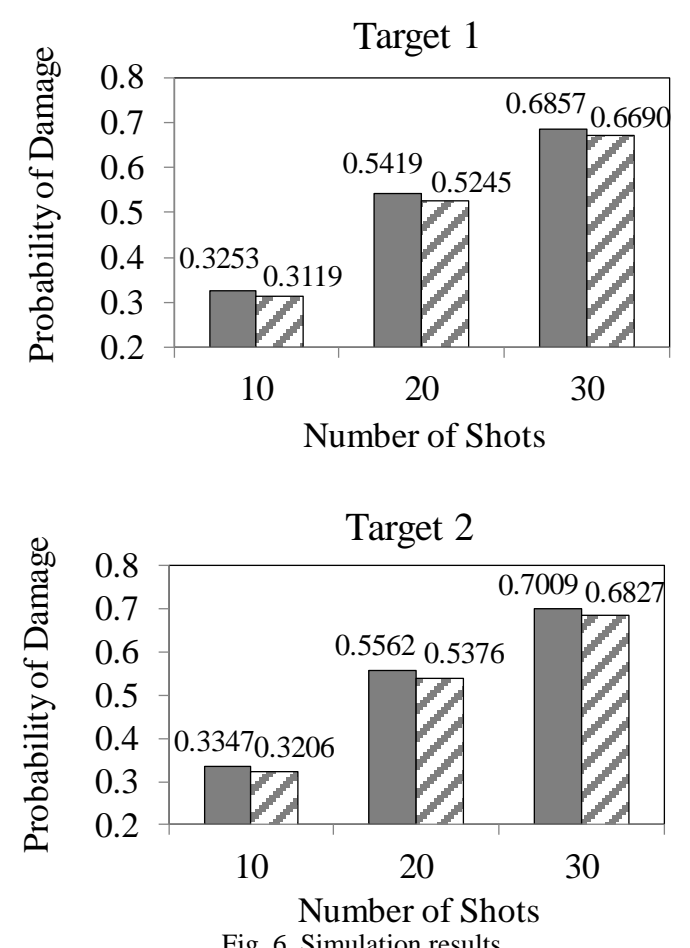

Fig. 6. Simulation results.

\begin{tabular}{|c|c|c|c|c|c|c|}
\multicolumn{7}{c|}{ TABLE I: RESUlTS OF SIMULATIONS ON TARGET 1 } \\
\hline \multirow{2}{*}{ \# shots } & \multicolumn{2}{|c|}{ Use Pk Matrix } & \multicolumn{2}{c|}{$\begin{array}{c}\text { Use Carleton } \\
\text { Function }\end{array}$} & \multicolumn{2}{c|}{ Difference } \\
\cline { 2 - 7 } & Mean & Std Dev & Mean & Std Dev & Mean & Std Dev \\
\hline 10 & 0.3253 & 0.1730 & 0.3119 & 0.1731 & 0.0134 & 0.0366 \\
\hline 20 & 0.5419 & 0.1760 & 0.5245 & 0.1799 & 0.0174 & 0.0424 \\
\hline 30 & 0.6857 & 0.1576 & 0.6690 & 0.1636 & 0.0167 & 0.0421 \\
\hline
\end{tabular}

TABLE II: RESULTS OF SIMULATIONS ON TARGET 2

\begin{tabular}{|c|c|c|c|c|c|c|}
\hline \multirow{2}{*}{ \# shots } & \multicolumn{2}{|c|}{ Use Pk Matrix } & \multicolumn{2}{c|}{$\begin{array}{c}\text { Use Carleton } \\
\text { Function }\end{array}$} & \multicolumn{2}{c|}{ Difference } \\
\cline { 2 - 7 } & Ave & Std Dev & Ave & Std Dev & Ave & Std Dev \\
\hline 10 & 0.3347 & 0.2057 & 0.3206 & 0.2123 & 0.0141 & 0.0579 \\
\hline 20 & 0.5562 & 0.2060 & 0.5376 & 0.2172 & 0.0186 & 0.0635 \\
\hline 30 & 0.7009 & 0.1808 & 0.6827 & 0.1937 & 0.0182 & 0.0602 \\
\hline
\end{tabular}

A paired t-test was performed in Minitab® R14 for each pair of the results of two simulations that employed Pk matrix and the Carleton function on the same number of shots and target sizes. Totally, 6 comparisons were performed. In all comparisons, $\mathrm{p}$-value was less than the significant level of 0.01 . So the null hypothesis was rejected and the alternative hypothesis could be accepted. It could be suggested that the difference between two means of the simulations is statistically significant. Fig. 7 shows the $99 \%$ confidence interval of the difference mean, as given in (4). In any case, the interval the mean of the differences did not include zero and was always positive. So the results of the simulations that 
employed Pk matrix were higher than the results of the same simulations that employed the Carleton function.

In addition, the histograms of the difference between the results in each pair of two simulation results were examined. The histograms of the simulations on target 1 are presented in Fig. 8 to Fig. 10. The histograms for the simulations on target 2 are presented in Fig. 11 to Fig. 13. All histograms show that the difference between the results in each run was normally distributed so the paired t-test should be valid.
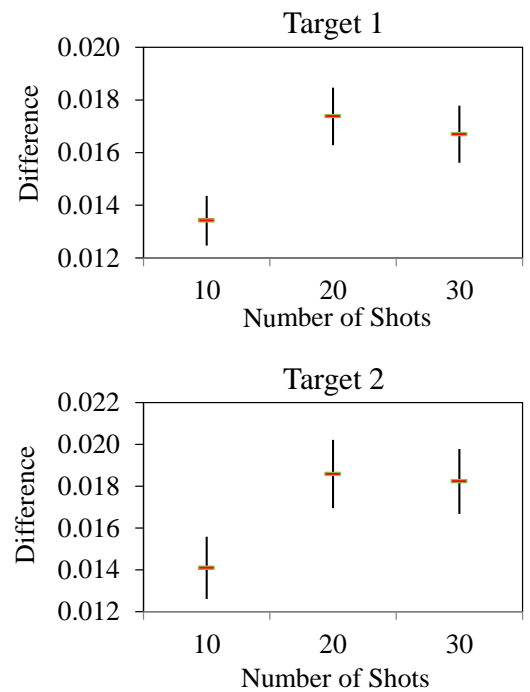

Fig. 7. The 99\% confidence interval of the difference between the results.

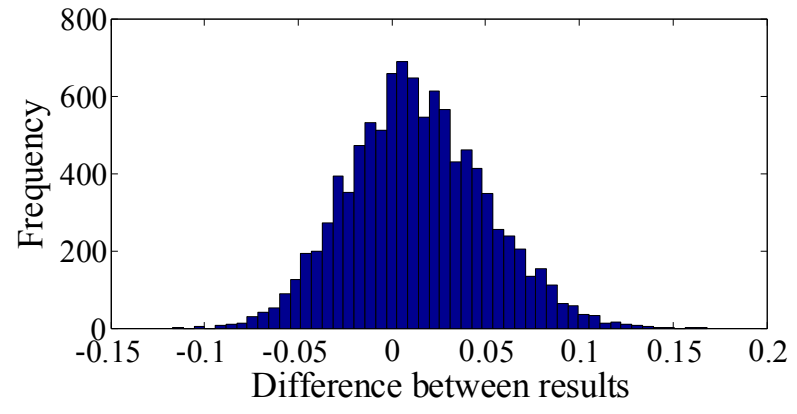

Fig. 8. Histogram of difference -10 shots against target 1 .

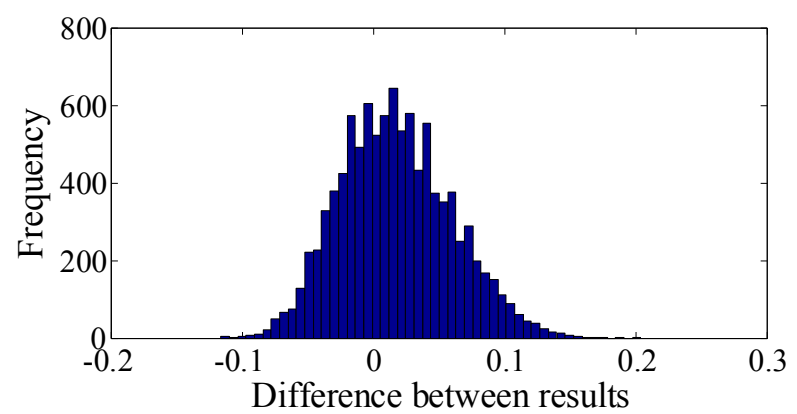

Fig. 9. Histogram of difference -20 shots against target 1 .

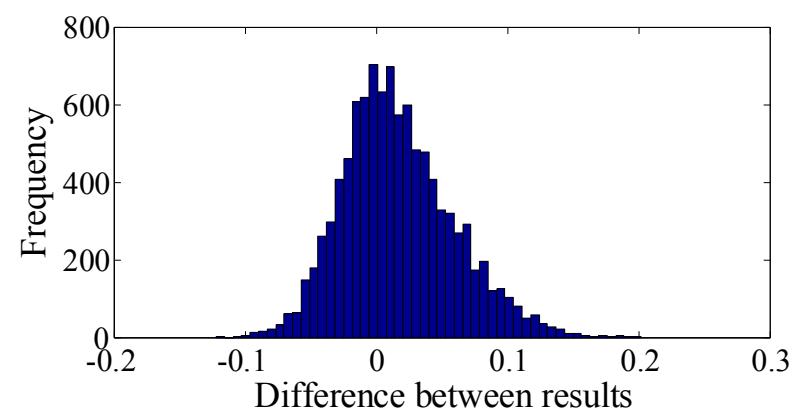

Fig. 10. Histogram of difference -30 shots against target 1 .

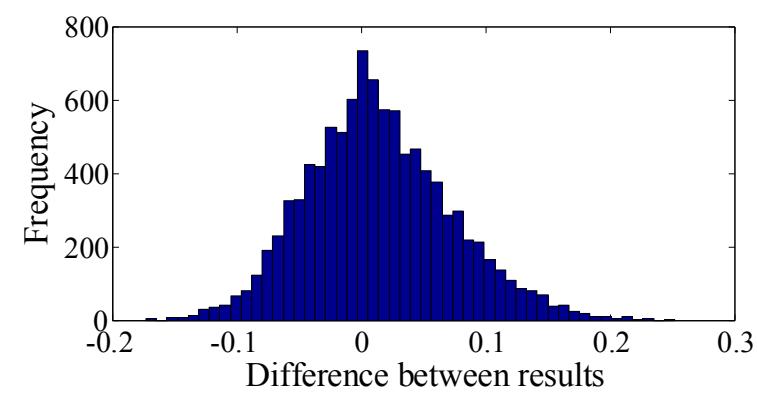

Fig. 11. Histogram of difference -10 shots against target 2 .

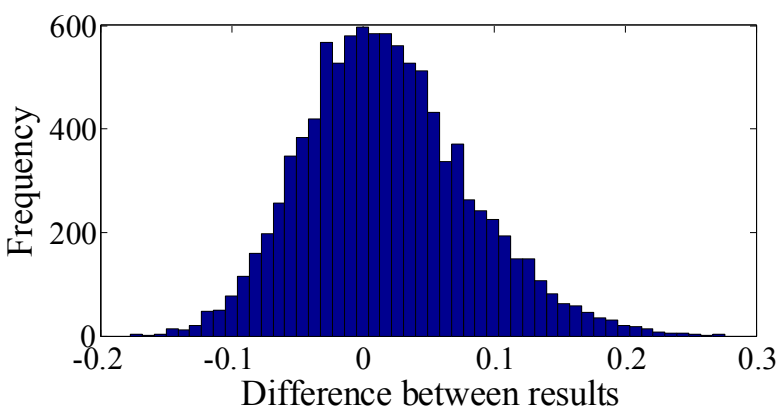

Fig. 12. Histogram of difference -20 shots against target 2 .

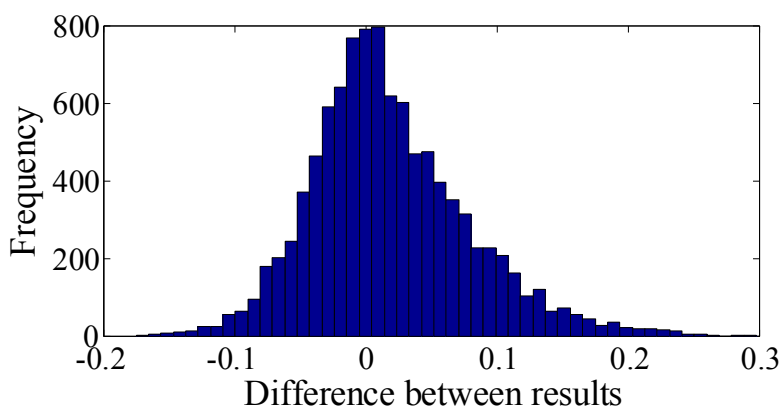

Fig. 13. Histogram of difference -30 shots against target 2 .

Very small differences can be detected as statistically significance in t-tests when the sample size are large, which is the case of Monte Carlo simulations. Therefore, it is important to investigate the effect size following the rejection of the null hypothesis. An effect size, Cohen's $d$ [13], was examined for all comparisons in the case study. The value of Cohen's $d$ was determined by (8), where $\bar{X}_{d i f f}$ is the mean and $S D_{\text {diff }}$ is the standard deviation of the differences.

$$
d=\frac{\bar{X}_{\text {diff }}}{S D_{\text {diff }}}
$$

Table III shows the value of Cohen's $d$ calculated in all six comparisons. The value of Cohen's $d$ ranges from 0.36 to 0.41 in target 1 cases and from 0.24 to 0.30 in target 2 cases. So the effect size in target 1 cases was larger than in target 2 cases. By a common guideline [13], the effect size is considered small for the simulations on Target 1 and between small and medium for the simulations on Target 2.

Based on Cohen's $d$ in presented Table III, the difference between the results of Monte Carlo simulations that employ $\mathrm{Pk}$ matrix and the results of the same simulations that employ the Carleton function was small. It could be recommended that Carleton function be used in Monte Carlo simulations instead of a non-simplified $\mathrm{Pk}$ matrix because it requires 
coarser grid and less computing time, which is a critical concern in a battlefield. However, users of the Carleton function should be aware that small difference does exist.

TABLE III: COHEN’S D VALUES

\begin{tabular}{|c|c|c|}
\hline \# Shots & Target 1 & Target 2 \\
\hline 10 & 0.367 & 0.243 \\
\hline 20 & 0.410 & 0.293 \\
\hline 30 & 0.396 & 0.303 \\
\hline
\end{tabular}

\section{CONCLUSION}

Two sets Monte Carlo simulations to determine the probability of damage were performed in a case study of multiple shots of unguided weapon against a uniform value area target. The first set of the simulations utilized a $\mathrm{Pk}$ matrix and the second set utilized the Carleton damage function, of which parameters were determined based on a $\mathrm{Pk}$ matrix. The simulations were performed for 10, 20, and 30 shots and 2 targets, of which the area is about 2.5 and 1.2 times of the weapon lethal area. Each simulation comprised 10000 runs. Both sets of simulations used the same impact points that were randomly generated in accordance with the weapon deliver accuracy. Totally, 6 paired t-tests were carried out and there was statistically difference between the simulation results that utilized the Pk matrix and the Carleton function. Furthermore, the effect size was investigated and it was suggested that the effect was small or almost medium in all comparisons. So it could be recommended that the Carleton be used in a simulation if the computing speed is concerned.

\section{ACKNOWLEDGMENT}

The authors would like to thank Dr.Ganchai Tanapornraweekit at Defence Technology Institute, Thailand, for his advice on the lethality of fragmenting warheads and Group Captain Chesda Kiriratnikom at Research and Development Centre for Space and Aeronautical Science and Technology, Royal Thai Air Force, for his suggestion on weapon effectiveness analyses.

\section{REFERENCES}

[1] A. R. Eckle and S. A. Burr, "Mathematical models of target coverage and missile allocation," DTIC: AD-A953517, Military Operations Research Society, USA, 1972.

[2] J. T. Klopcic, "A comparison of damage functions for use in artillery effectiveness codes," BRL-MR-3823, Ballistic Research Laboratory, Aberdeen Proving Ground, MD, USA, 1990.
[3] J. S. Przemieniecki, Mathematical Methods in Defense Analyses, 3rd ed., AIAA Education Series, USA, 2000.

[4] J. W. Kim, C. Lee, and B. R. Cho, "Using a Rayleigh-based circular lethality coverage for naval surface fire support," presented at Industrial Engineering Research Conference, FL, USA, May 19-21, 2002.

[5] M. R. Driels, Weaponeering: Conventional Weapon System Effectiveness, 2nd ed., AIAA Education Series, USA, 2013.

[6] C. M. Anderson, "Generalized weapon effectiveness modeling," Master's Thesis, Naval Postgraduate School, Monterey, CA, USA, 2004.

[7] Joint Technical Coordinating Group for Munitions Effectiveness Publications, AR 25-35/AFI 10-411/MCO 5600.43B/OPNAVINST $5600.23,1996$.

[8] N. Metropolis, "The beginning of the Monte Carlo method," Los Alamos Science, issue 15, pp. 125-130, 1987.

[9] R. E. Roger, "Stan Ulam, John von Neumann, and the Monte Carlo method," Los Alamos Science, issue 15, pp. 131-137, 1987.

[10] M. R. Driels and Y. S. Shin, "Determining the number of iterations for Monte Carlo simulation of weapon effectiveness," Technical Report, Naval Postgraduate School, Monterey, CA, USA, 2004.

[11] R. Y. Rubinstein and D. P. Kroese, Simulation and the Monte Carlo Method, 2nd ed., John Wiley and Sons, USA, 2008.

[12] A. Black, J. F. Mahoney, and B. D. Sivazlian, "Estimation of the weapons parameters and their variances in the Carleton damage function," Variability of Measures of Weapons Effectiveness, vol. 6 , AFATL-TR-84-92, Air Force Armament Laboratory, USA, 1985.

[13] J. Cohen, Statistical Power Analysis for the Behavioral Sciences, 2nd ed., Lawrence Erlbaum Associates, USA, 1988.

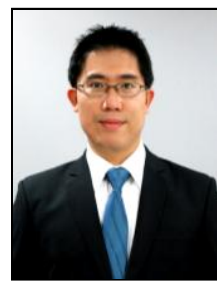

Pawat Chusilp received his B.S. in mechanical engineering from Chulalongkorn University, Thailand in 1999, M.S. and Ph.D. in mechanical engineering from University of Southern California, USA in 2001 and 2004 respectively. He joined Defence Technology Institute, Thailand in 2009 and is currently a researcher in aeronautical engineering laboratory. His research interests are in aerodynamics, trajectory simulations, and target coverage.

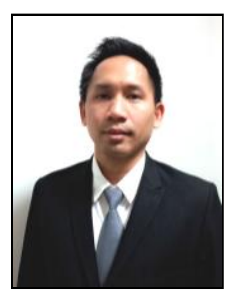

Weerawut Charubhun earned his B.S. in mechanical engineering from Kasetsart University, Thailand in 1999 and M.S. in mechanical engineering from University of Sydney, Australia in 2002. He joined Defence Technology Institute, Thailand in 2009 and is currently a researcher in aeronautical engineering laboratory. His research interests are in aerodynamics, computational fluid dynamics, and trajectory simulations.

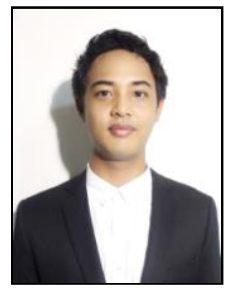

Pattadon Koanantachai received his B.S. in aerospace engineering and M.S. in mechanical engineering from King Mongkut's University of Technology North Bangkok, Thailand in 2010 and 2014 respectively. He joined Defence Technology Institute, Thailand in 2014 and has been working as a researcher in aeronautical engineering laboratory. His research interests are in aircraft designs, computational fluid dynamics, and composite airframe structures. 